\title{
TTR
}

Traduction, terminologie, re?daction

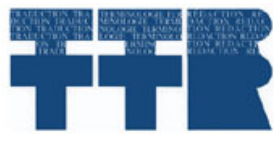

\section{Migrations littéraires : Maryse Condé et Emily Brontë}

\section{Anne Malena}

Volume 13, numéro 2, 2e semestre 2000

Les Antilles en traduction

The Caribbean in Translation

URI : https://id.erudit.org/iderudit/037411ar

DOI : https://doi.org/10.7202/037411ar

Aller au sommaire du numéro

\section{Éditeur(s)}

Association canadienne de traductologie

ISSN

0835-8443 (imprimé)

1708-2188 (numérique)

Découvrir la revue

Citer cet article

Malena, A. (2000). Migrations littéraires : Maryse Condé et Emily Brontë. TTR, 13(2), 47-74. https://doi.org/10.7202/037411ar

\section{Résumé de l'article}

Migrations littéraires : Maryse Condé et Emily Brontë

— En tant que ré-écriture de Wuthering Heights (1847) d'Emily Brontë, La Migration des coeurs (1995) de Maryse Condé transpose le classique anglais dans un contexte antillais marqué par la violence colonialiste et

l'hétérogénéité. Ce procédé de ré-écriture est un procédé de traduction dans le sens large du terme parce que l'improvisation à laquelle se livre Condé maintient un lien métonymique avec l'original tout en fonctionnant de façon indépendante. À son tour, la traduction anglaise du roman de Condé, Windward Heights (1998), suit ces pistes brouillées mais, par manque de stratégies conséquentes de traduction, compromet l'élan créateur de Condé en rapprochant son texte trop près de celui de Brontë. Cette étude montrera que ces mouvements de migration littéraire impliquent que l'écriture s'appuie sur des procédés de traduction et que la ré-écriture maintient une difficile relation métonymique avec l'original en lui rendant hommage tout en le transformant.
Tous droits réservés (C) TTR: traduction, terminologie, rédaction — Les auteurs, 2000
Cedocument est protégé par la loi sur le droit d'auteur. L'utilisation des services d'Érudit (y compris la reproduction) est assujettie à sa politique d'utilisation que vous pouvez consulter en ligne.

https://apropos.erudit.org/fr/usagers/politique-dutilisation/ 


\title{
Migrations littéraires : Maryse Condé et Emily Brontë
}

\author{
Anne Malena
}

À Emily Brontë
qui aurait, je l'espère, agréé cette lecture de son chef-d'œuvre.
Honneur et respect!

En lançant ce salut typiquement caribéen à l'écrivaine anglaise, Maryse Condé signale clairement dans la deuxième épigraphe en tête de $L a$ Migration des cours (1995) qu'il s'agit d'une ré-écriture de Wuthering Heights (1847) ou sans doute, étant donné la formation avant tout française de l'auteure guadeloupéenne, des Hauts de Hurle-Vent (1929) ${ }^{1}$. La traduction anglaise du roman de Condé, Windward Heights (1998), suit donc les pistes brouillées d'un original lui-même issu d'un processus de créolisation et de traduction.

En voulant démêler quelques-uns des fils de ce fascinant intertexte, cette étude se penchera sur la problématique de ce que je nomme la double écriture de Condé ${ }^{2}$. Dans un premier temps, il sera

1 Selon le quatrième de couverture, Maryse Condé aurait " toujours rêvé d'adapter à l'univers caraibe le roman d'Emily Brontë ». Cette dernière, morte en 1848 , ou un an après la parution de son roman, n'a pas vécu assez longtemps pour produire d'autres cuvres. Il faut pourtant signaler que, enfant, Emily Brontë inventait des histoires avec ses deux sœurs, Anne et Charlotte, et son frère Branwell et les représentait en jouant avec les soldats en bois de son frère. Par la suite, ils ont mis par écrit certaines de ces histoires et en ont fait des petits " livres ». Anne et Emily ont ainsi écrit la saga de " Gondal », une île mythique, où elles mettaient en scène un royaume féministe et royaliste (Peterson, 1992, p. 6).

${ }^{2}$ Je ne traiterai pas directement ici le danger inhérent à tout intertexte qui est d'être interprété comme plagiat. C'est en effet une accusation que porte Roger 
démontré que, parce qu'il est une ré-écriture, le roman illustre le principe élaboré par Gayatri Spivak selon lequel le sujet colonisé ne peut jamais se départir de l'impérialisme qui l'a créé, à moins d'en faire exploser les structures :

No perspective critical of imperialism can turn the Other into a self, because the project of imperialism has always already historically refracted what might have been the absolutely Other into a domesticated Other that consolidates the imperialist self. (Spivak, 1985 , p. 253)

Les personnages de Condé démontrent en effet qu'ils appartiennent tous au même système et que, malgré leur lutte pour la libération, leur humanité s'en trouve à jamais menacée. Condé parvient à faire exploser le monde fermé du roman de Brontë et à révéler les forces obscures qui le sous-tendaient comme étant la conséquence de la logique impérialiste, avide de pouvoir et de richesses. Ce procédé de ré-écriture est un procédé de traduction dans le sens large du terme parce que l'improvisation à laquelle se livre Condé maintient un lien métonymique avec l'original. Maria Tymoczko, en démontrant l'aspect métonymique de la relation entre l'oralité des mythes et la littérature, souligne l'importance d'analyser des traductions pour acquérir une meilleure compréhension de la littérature en général et, vice versa, d'analyser des exemples de ré-écriture pour mieux comprendre les procédés de traduction. C'est donc dans cette double perspective que je propose de placer ce travail.

Dans un deuxième temps, l'analyse de la traduction anglaise de La Migration des coeurs montrera qu'elle a un effet malheureux : en rapprochant trop le roman antillais de son modele anglais, elle risque d'en arrêter la dynamique régénératrice dans laquelle la ré-écriture de Condé l'a fait s'engager en lui assurant une survie des plus intéressantes qui nous invite à le relire à partir d'un point de vue périphérique et colonial.

Little contre Condé en relevant un passage calqué sur Ourika (Little, 1999, p. 72). Selon moi, cet usage du texte de Madame de Duras rentrerait plutôt dans la logique que je présente dans cette étude et qui est conforme au principe poststructuraliste que l'on ne fait jamais que redire ce qui a dẻjà été dit et que toute écriture est ré-écriture (voir Tymoczko, 1999, p. 41). Comme on le verra, ce principe est aussi celui de la traduction, génératrice de différences riches en signification. 


\section{Double écriture}

Selon Gayatri Spivak, la portée féministe et postcoloniale du roman de Jean Rhys, Wide Sargasso Sea, est limitée par le fait que les personnages recréés dans une perspective antillaise ne peuvent fonctionner qu'en tant qu'allégorie de la violence exercée par le colonialisme sur l'épistémè des cultures qu'il domine. Ainsi, la réincarnation de Bertha, Antoinette, acquiert sa propre subjectivité mais finit quand même par s'immoler elle-même, perpétuant ainsi la logique impérialiste du modèle de Charlotte Brontë, Jane Eyre (Spivak 1985, pp. 250-251). En transposant Wuthering Heights du Yorkshire au début du dix-neuvième siècle aux Antilles à la fin du dix-neuvième siècle, Maryse Condé fait écho à la tentative de Rhys en inscrivant les dichotomies brontëennes entre bien/mal, humanité/bestialité, éducation/ignorance dans le contexte socio-historique explicite de la fin des grandes plantations et de la prise de pouvoir par les Noirs ${ }^{3}$. Cette " performance " antillaise du roman de Brontë fonctionne aussi en tant qu'allégorie de l'impérialisme, mais va plus loin dans sa critique et dans sa volonté de représenter les relations de pouvoir à l'œuvre dans une société issue du colonialisme et de démontrer qu'il s'agit d'une société malade à jamais marquée par la cupidité qui fut à l'origine de sa création. Contrairement à Wide Sargasso Sea, La Migration des caurs ne s'affiche pas comme une correction du roman d'Emily Brontë, ni comme un véhicule privilégié de la représentation antillaise d'un seul personnage, mais comme sa ré-écriture et son développement créole. Les fils sémiotiques, que Condé ramasse au fond du Yorkshire pour en tisser son propre ouvrage en Guadeloupe, sont noués et dénoués, coupés et entrecoupés, transformés et colorés par l'histoire politique, culturelle et littéraire au cours de leur traversée de l'Atlantique, métaphore littéraire qui n'est pas sans rappeler le passage des cultures africaines, européennes ou indiennes aux cultures créoles. Il y a donc lieu de s'interroger sur les signes plausibles qui, dans le roman de Brontë, ont retenu l'attention de Condé et inspiré cette ré-écriture.

Un signe, d'après Peirce, en génère d'autres selon certains aspects de l'objet qui est représenté. Il se crée ainsi un réseau dynamique de chaînes sémiotiques qui produisent ce que Peirce appelle

\footnotetext{
${ }^{3}$ Mouvement tout à fait parallèle, bien sûr, à la transposition effectuée par Jean Rhys à l'époque de l'émancipation en Jamaïque.
} 
un objet immédiat alors que la totalité de l'objet dynamique, ou l'objet réel, reste juste au-delà du champ sémiotique ou hors de portée du processus d'interprétation. En ce qui concerne la transposition de Wuthering Heights vers les Antilles, nous verrons que les signes se multiplient parce que la narration se pluralise, créant ainsi un univers fictif polyphonique dans lequel évoluent des personnages inextricablement liés entre eux par des liens socio-historiques et culturels $^{4}$. Chaque personnage poursuit donc sa dynamique d'un champ sémiotique à l'autre, ce qui a pour effet de créoliser le réseau auquel ils appartiennent. Pourtant, avant de permettre à ses personnages d'évoluer sur la scène antillaise, Condé doit effectuer une transposition temporelle.

Alors que la narration de Wuthering Heights débute en 1801 pour se terminer un an plus tard et relate l'histoire de deux familles à la fin du XVIII ${ }^{\mathfrak{e}}$ siècle, l'intrigue de La migration des coeurs ne peut que commencer après l'abolition de l'esclavage dans les colonies (1848 pour les colonies françaises et 1876 pour les colonies espagnoles), c'est-à-dire à la fin du XIX ${ }^{e}$ siècle. Les événements marquant les deux familles parallèles antillaises se prolongent donc jusqu'au début du $\mathrm{XX}^{\mathrm{e}}$ siècle, au cours d'une violente période de transition qui signifiait la fin des grandes plantations possédées par les békés, les blancs, sans pour autant en effacer les conséquences d'ordre social et symbolique. Afin de se remettre en tête l'intrigue de Wuthering Heights et de mieux comprendre le processus de créolisation auquel la soumet Condé, il est utile de comparer les généalogies des deux familles dans chacun des romans ${ }^{5}$. Cette comparaison révèle plusieurs principes de traduction et d'adaptation, en particulier en ce qui concerne l'onomastique, les descriptions physiques des lieux et des personnages, le décuplement des personnages et la transformation des liens familiaux d'un texte à l'autre (pour les détails, voir tableau en appendice).

${ }^{4}$ Pour plus de détails sur le caractère triadique du signe, voir Peirce, 1977, p. 83 et pour l'application de ce concept à un autre roman de Condé, Traversée de la mangrove, voir Malena, 1999, pp. 67-91.

${ }^{5}$ Dans une lecture marxiste du roman de Brontë, Terry Eagleton suggère que la généalogie n'est pas sans lien avec la structure sociale : « familial relations at once provide the substance of antagonism and mold that substance with intricate shape, precipitating a highly integrated form for the very stuff of struggle and desintegration. The genealogical structure, moreover, allows for a sharply dialectical relation between the 'personal' and 'impersonal' » (Eagleton, 1992, p. 400). 
L'élément central à cette transposition est Heathcliff parce qu'il est le personnage étranger qui vient s'immiscer entre les deux familles et en provoquer les alliances et les mésalliances. Condé le renomme Razyé, un prénom tiré du terme désignant l'herbe de la savane antillaise et donc rattaché au paysage comme celui de Heathcliff. Ce nom évoque également le mot algérien de razzia qui désigne l'acte de piller et de saccager, ou de razzier les récoltes d'une tribu, une activité à laquelle se livre Razyé en mettant le feu aux champs de canne des békés. Bien que sa vengeance soit personnelle et dirigée contre le mari de Cathy, elle acquiert une dimension collective et politique puisque Razyé la met au service de la montée au pouvoir des Noirs. Dans la même veine, l'apparence des personnages acquiert aussi une teneur raciale, à peine sous-jacente chez Brontë. Quant à la prolifération de chaque branche de l'arbre généalogique, elle appartient également à un contexte antillais où les familles étaient nombreuses et les relations extra-maritales fréquentes. Ainsi, Aymeric de Linsseuil, donc la contrepartie d'Edgar Linton, se remarie après la mort de Cathy, probablement parce qu'il faut une mère à ses trois orphelins, et engendrera quatre enfants de plus dont trois survivront. Razyé, pour sa part, engendre cinq enfants, six si l'on compte Cathy qui est très probablement sa fille. Cette abondante progéniture le distingue de Heathcliff qui avait pour fils unique le maladif Linton, lequel meurt peu après son mariage forcé avec Cathy. Enfin, la naissance d'Anthuria, fille du fils de Razyé et de la fille de Cathy, prolonge cette généalogie antillaise et peut être vue soit comme la perpétuation du cycle tragique dépeint dans La migration des caurs soit comme la possibilité de sa rupture, en tout cas comme une promesse de vie.

Wuthering Heights représentait un monde marginal de la petite noblesse rurale divisé par la violence binaire qui oppose deux stéréotypes : le premier étant le milieu sauvage et bestial de Wuthering Heights et le second le domaine raffiné et aristocrate de Thrushcross Grange. Les personnages vivaient dans une isolation complète; ils n'entretenaient apparemment aucune relation sociale en dehors des membres de la famille et les serviteurs semblaient jouir de plus de mobilité que les maîtres. Heathcliff était un perturbateur venu troubler ce calme apparent; son arrivée précipita dans les deux familles un cercle vicieux de désirs inassouvis et de passions vengeresses qui ne serait brisé qu'après sa mort. M. Earnshaw l'avait rapporté sous son manteau en cadeau à ses enfants, Hindley et Cathy, après l'avoir découvert dans le port de Liverpool. En le présentant à sa famille, il le 
décrivit comme « un présent de Dieu, bien qu'il soit presque aussi noir que s'il sortait de chez le diable » (Brontë, 1992, p. 55) ${ }^{6}$. Assez grand pour marcher et parler, bien qu'il se bornât " à baragouiner indéfiniment quelque chose que personne ne put comprendre » (Brontë, 1992, pp. 55-56), l'enfant se fit qualifier de " petit bohémien » (Brontë, 1992, p. 56) ${ }^{8}$ par la maîtresse de maison, détesté par Hindley, mais adoré par Cathy.

Cet être exotique venu de la mer, que ses origines obscures rendent dangereux, est peut-être le produit d'une agitation sociale extra-diégétique, un rejeton irlandais ou hybride d'éléments marginaux'. À l'époque où Emily Brontë écrivait, le climat social était loin d'être harmonieux : l'avènement et la rapide croissance de l'industrialisation mettaient des centaines de travailleurs en textile au chômage et les incitaient à brûler les métiers à tisser mécanisés; le mouvement prolétaire chartiste organisait d'énormes manifestations dans les villes pour réclamer le droit de vote et des milliers d'Irlandais se réfugiaient dans les ports anglais pour échapper à la famine qui fit plus de 700000 morts (voir Peterson, 1992, p. 12). Au sein du monde statique du Yorkshire, Heathcliff représente le signe d'une transformation possible, à la fois redoutée et peut-être secrètement

${ }^{6}$ Les citations françaises sont tirées de la traduction de Delebecque. Les citations correspondantes en anglais seront mises en note: " a gift of God; though it is as dark almost as if it came from the devil » (Brontë, 1959, p. 40).

${ }^{7}$ « repeated over and over again some gibberish that nobody could understand » (Brontë, 1959, p. 41).

${ }^{8}$ " gypsy brat » (Brontë, 1959, p. 51).

${ }^{9}$ On pourrait se demander aussi s'il est l'enfant illégitime de M. Earnshaw, ce qui expliquerait l'aversion que sa femme ressent immédiatement pour lui. Cette supposition paraîtrait presque naturelle dans le contexte antillais créé par Condé vu qu'il était fréquent que les hommes entretiennent des concubines. Le texte de Condé y fait d'ailleurs référence dans le passage qui précède la découverte de Razyé : "Les gens disaient que pendant ses séjours à la Pointe Hubert Gagneur en profitait pour faire la fête avec Amélie, une capresse qui habitait sur le Morne-à-Cayes et qui était sa maîtresse depuis des temps et des temps ” (Condé, 1995, p. 26). Il faut également noter que, à la lumière du texte de Condé, le port de Liverpool chez Brontë acquiert une dimension coloniale et esclavagiste vu qu'il est connu maintenant que Liverpool était un des ports impliqués dans la traite des esclaves (voir Thomas, 1997, pp. 243-284). 
souhaitée. Il fait ainsi figure de dangereux séducteur : son amour et sa loyauté pour Cathy sont tellement extrêmes qu'ils doivent être condamnés par la société et ne peuvent être égalés que par la cruauté qu'il témoigne envers Isabelle, son propre fils, Edgar Linton et la fille de Cathy. Il est clair que cette cruauté est le résultat du mauvais traitement que Heathcliff a souffert aux mains de Hindley après la mort de Mr. Earnshaw : il a été déclassé, mis au rang des palefreniers et maintenu en dehors des droits, des privilèges et des liens de la famille.

Pourtant, l'absence dans Wuthering Heights d'un ancrage socio-historique explicite fait que le désir de vengeance de Heathcliff est individualisé à outrance et ne relève plus que de la psychologie. Il devient une force cruelle et néfaste, pour ainsi dire inhumaine, qu'il s'agit d'éliminer. Le dénouement heureux, à savoir le mariage de Cathy et de Hareton, neutralise la force révolutionnaire qu'il aurait pu avoir et ne fait rien pour problématiser le binarisme qui s'opère entre la classe dominante des Linton et la classe laborieuse des Earnshaw. Le Yorkshire est un lieu périphérique où sont reproduites et où demeurent intactes les structures du capitalisme.

Par contraste, l'agitation sociale que Brontë a maintenue au niveau de l'implicite fournit à Condé une toile de fond pour son roman. La colonie française de la Guadeloupe, où elle fait émigrer les coeurs, fait éclater le binarisme et en dissémine les fragments à travers les classes, les races et les îles de l'archipel de la Guadeloupe, de Cuba et de la Dominique. Razyé n'en est pas non plus un élément externe mais bel et bien antillais : il partage ses origines obscures avec des millions d'autres habitants des îles et, comme eux, il est un être venu de la mer.

Condé reprend une technique narrative qui l'avait bien servie dans Traversée de la mangrove et qui est inspirée de Faulkner, en particulier dans As I Lay Dying. Cette technique consiste à multiplier les narrateurs et les points de vue pour créer un univers fictif polyphonique et irréductible. La double narration que Brontë avait utilisée pour répondre aux exigences du roman victorien fait place à un tissage pluriel et mouvant de voix narratives. Ainsi, Mr. Lockwood, le touriste venu se reposer au Yorkshire, disparaît et Nelly Dean, la servante fidele des deux familles et substitut maternel des deux Cathy, se divise en au moins six personnages, descendantes d'esclaves ou de 
Zindiens $^{10}$ : Nelly Raboteur qui s'occupe de Cathy jusqu'à son mariage, Lucinda Lucius qui s'en occupe après son mariage, mabo Julie qui a élevé Irmine de Linsseuil, Sanjita, d'origine indienne, qui s'occupe d'un autre domaine des Linsseuil, mabo Sandrine qui élève la deuxième Cathy et Ada qui raconte la grossesse de cette dernière et sa mort à la Dominique. D'autres personnages viennent insérer leur récit dans l'histoire qui commence à Cuba pour passer en Guadeloupe, puis à Marie-Galante, à la Dominique et se termine en Guadeloupe. Ce partage discursif permet d'offrir une vue kaléidoscopique de la société antillaise et d'insister sur la nature fluide des catégories qui la composent. Tous ces personnages, aussi distincts soient-ils d'un point de vue économique et racial, participent néanmoins à la même histoire, celle de la Guadeloupe et celle de la Caraíbe. Une histoire plurielle qui résiste à tout effort, selon Benítez-Rojo, de rendre fixe l'image furtive de son être collectif (Benitez-Rojo, 1992, p. 1).

En voulant saisir la dynamique culturelle du méta-archipel que forment les îles de la Caraïbe, Antonio Benitez-Rojo se sert de la figure centrale de la plantation qui, par sa production sucrière, fonctionne en tant que machine nourricière de l'avidité capitaliste des métropoles (Benítez-Rojo, 1992, p. 38). Parce que les colonies antillaises ont été créées de toutes pièces pour subvenir à la demande de sucre des métropoles, elles se sont développées de façon chaotique et hétérogène suivant les caprices de l'économie européenne et les mélanges ethniques de leur main-d'œuvre. D'où la mouvance des significations culturelles de la région et le besoin de préciser que, lorsqu'on parle de syncrétisme culturel ou religieux, par exemple, on parle du produit changeant d'un mouvement perpétuel de définition, d'appropriation et de transformation. Ainsi, les croyances africaines coexistent avec les croyances des premiers habitants, Caraibes ou Arawaks, et celles venues d'Europe, des Indes ou de la Chine et chacune d'entre elles s'adapte et se transforme au contact des autres. Il s'agit donc d'une culture de chaos qui charrie, dans sa fluidité, la violence de ses origines, la multiplicité de son histoire, la fragmentation de sa géographie et l'hétérogénéité de ses cultures et de ses langues.

${ }^{10}$ Terme créole utilisé dans le texte et qui se réfère aux individus d'origine indienne. Au terme de l'esclavage, des milliers de travailleurs agricoles ont quitté l'Inde pour venir travailler dans les plantations antillaises. 
La transposition des Hauts de Hurle-Vent dans la Caraïbe permet ainsi de créer un univers humain animé par les mêmes passions que celles qu'on trouvait au fond du Yorkshire au début du XIX ${ }^{e}$ siècle, mais un univers où les vents du monde entier sont venus s'engouffrer (la signification même du nom de l'Engoulvent qui est aussi un lieu sans issues), jetant ensemble Caraïbes, Arawaks, Européens, Africains et Indiens. Le mouvement isentropique dans lequel Maryse Condé entraîne le texte d'Emily Brontë mènera à des conclusions différentes parce que le contexte du roman est d'ores et déjà celui d'une société hybride dont Razyé est à la fois membre intégral et représentation du malaise qui l'agite, le signe de la violence de sa genèse, de la révolte de son présent et de l'incertitude de son avenir.

Ainsi placé sous le signe du chaos, le roman s'ouvre à Cuba sur une scène décrivant la procession du jour des Rois, vingt ans après l'abolition de l'esclavage, que l'Espagne ne décréta qu'en 1876. Le cortège est décrit comme une activité carnavalesque qui permet aux cultures africaines, organisées en cabildos, de s'exhiber sous l'œil bénévole du gouverneur :

Derrière Melchior [le babalawo, prêtre de Chango, que tout le monde respecte mais qui se fera néanmoins assassiner ce soir-là] venaient les Congos et les Lucumis en bleu et noir, les Araras en jupes de raphia ébouriffé, les joues striées de balafres tracées au fer rouge ou au couteau, et enfin les Mandingues tellement élégants dans leurs pantalons bouffants, la tête enturbannée de mètres et de mètres de toile indigo. (Condé, 1995, p. 11)

Selon Benítez-Rojo, l'africanisation considérable de la culture cubaine s'explique en partie par la présence d'un pourcentage élevé de noirs libres (en 1774, 20,3\% de la population entière) qui ont été des agents importants d'acculturation (Benítez-Rojo, 1992, pp. 121-122). D'emblée, plusieurs détails de la description de Condé font allusion à l'hétérogénéité de cette culture et à son manque d'harmonie. Le cortège se termine en débandade suggérant d'autres forces en jeu que celles de la culture et de la religion organisées :

Un désordre de femmes et d'enfants de toutes les couleurs de l'arcen-ciel, depuis le noir kongo jusqu'au blanc à s'y tromper, galopait n'importe comment. [...] De temps en temps, Melchior ne pouvait s'empêcher de se retourner et de jeter des regards de colère vers la queue de la procession. Personne n'y faisait attention et la bacchanale continuait. (Condé, 1995, p. 12) 
Ce désordre est aussi l'affirmation de la vie, un défi à toute tentative de définition et l'expression irrépressible de la culture du chaos, culture de résistance qui trouve moyen de se manifester dans toutes les failles et interstices du système social et politique qu'elle caractérise.

Ce choix de mise en scène pour le début du roman offre d'emblée un contexte socio-historique marqué par l'agitation politique d'une société en train de se débarrasser du joug de quatre siècles d'asservissement. Il s'agit pourtant d'une agitation dont le succès est menacé puisque mention est faite de la mort du révolutionnaire José Marti et de l'intervention imminente de la part des États-Unis dans la guerre d'indépendance. Razyé, en tant que signe, incarne cette agitation et, par son retour en Guadeloupe, la transportera dans l'île française où il reste beaucoup à faire pour renverser les structures coloniales. Mais Razyé est un anti-héros : il est peint comme un être maudit et solitaire qui, après avoir perdu Cathy, fait figure de mauvais esprit, dangereux même pour les sorciers les plus puissants. La couleur noire qui caractérisait déjà Heathcliff" ${ }^{11}$, place Razyé au bas de l'échelle sociale et l'empêche à jamais d'être accepté au rang de l'humanité. Cathy, qui est mulâtre, rejette Razyé parce que, sans fortune et avec sa peau noire ashanti, il représente l'antithèse de l'acceptation sociale qu'elle souhaite. Elle confie à sa mabo Nelly Raboteur :

Je ne pourrai jamais, jamais me marier avec Razyé. Ce serait trop dégradant. Ce serait recommencer à vivre comme nos ancêtres, les sauvages d'Afrique! (Condé, 1995, p. 20)

Il est suggéré que Melchior se fait tuer par « Jaruco, un mauvais nègre, un joueur de couteaux qui ne respectait rien, pas même les morts, et que l'on appelait le Détrousseur " (Condé, 1995, p. 17), parce que Razyé avait exigé de lui d'être initié aux secrets réservés aux babalawo et que Chango n'a pas toléré cette infraction. Après la mort de Cathy, Razyé

" Dans la traduction française, le mot « nègre " est même utilisé par Nelly Dean au cours d'un dialogue avec Heathcliff qui se plaint de ne pas pouvoir égaler Edgar Linton aux yeux de Cathy. Il dit: “ En d'autres termes, il faut que je souhaite d'avoir les grands yeux bleus et le front uni d'Edgar Linton... ". À quoi, Nelly répond: " Un bon cceur vous aidera à avoir une bonne figure, mon garçon, fussiez-vous un vrai nègre " (Brontë, 1992, p. 82). Bien que cette traduction de l'expression « regular black », utilisée par Brontë $(1959$, p. 60), soit répréhensible, il est possible qu'elle ait influencé la lecture qu'en a fait Condé. 
continuera à vouloir prendre contact avec les egun et provoquera ainsi, indirectement, la mort de plusieurs sorciers célebres et puissants.

Comment interpréter cette force maléfique et surnaturelle dont le personnage de Razyé est empreint? Razyé est littéralement le produit réifié de la société esclavagiste qui s'est construite sur le corps des esclaves sans se soucier des conséquences et sans prévoir que, même au plus profond de l'abjection, un être humain ne perd jamais la capacité d'aimer, de souffrir et de se battre. Dans ce sens, Razyé est un prototype de révolte qui, au cours du roman, se fait peu à peu supplanter par une prise de conscience collective et un mouvement social cherchant à renverser la structure du pouvoir. Avant de retourner en Guadeloupe, il fait fortune à Cuba et il s'instruit, comme le démontre le fait qu'il lit Bug-Jargal sur le bateau qui le ramène. Ce choix de lecture ajoute aussi à son aura révolutionnaire puisque Victor Hugo a choisi la révolution de St-Domingue (1791-1804) comme contexte de son roman. La malédiction de l'esclavage marque donc chacun des personnages et se manifeste dans leur comportement.

Le père de Cathy, le mulâtre Hubert Gagneur, sauve Razyé d'un cyclone et le ramène à l'Engoulvent. Contrairement à Heathcliff, le mal qui marque l'enfant lui est extérieur : "C'est sûrement les mauvais esprits, cachés dans les vents du cyclone, qui l'ont amené de notre côté " note son père adoptif (Condé, 1995, p. 28). Par son apparence, Razyé incarne aussi l'hybridité antillaise : " Un enfant de sept ou huit ans, sale et repoussant, complètement nu, garçon, et, croyez-moi, le sexe bien formé, nègre ou bata-zindien. Sa peau était noire, mais ses cheveux bouclés s'emmêlaient jusqu'au milieu de son dos " (Condé, 1995, p. 28). La description faite par la servante Nelly Raboteur charrie les préjugés raciaux profondément assimilés par les membres d'une société esclavagiste, la prétendue puissance sexuelle des noirs et la dégradation associée avec quelqu'un de couleur foncée. La façon dont Hubert Gagneur traite l'enfant trouvé renforce ces stéréotypes :

Il lui apprenait avec toutes qualités de gros mots, les biguines les plus obscènes. Il se tordait de rire à le voir danser en frétillant du bonda ou en pointant son sexe. (Condé, 1995, p. 29)

Ce père adoptif reproduit ainsi dans sa façon d'agir le système de valeurs des planteurs blancs qui faisaient danser les esclaves pour leur propre amusement. Ces valeurs sont les mêmes qui ont poussé Cathy à 
épouser Aymeric et celles desquelles Razyé va tenter de se venger en lançant une campagne de destruction contre les békés.

De retour de Cuba, Razyé se met au service d'un maire socialiste (une allusion au parti fondé par un Noir, Hégésippe Légitimus, et qui s'empara de plusieurs mairies en 1896) afin de ruiner Aymeric de Linsseuil, le dernier grand propriétaire foncier de GrandeTerre. L'industrialisation a rendu possible le maintien de l'économie de plantation, même si les grandes plantations ont disparu, par l'installation d'usines liées par contrat à des groupes de planteurs. Razyé, en brûlant ses champs de canne, parvient à ruiner Aymeric, forçant ainsi la jeune Cathy à prendre un poste d'institutrice à MarieGalante où Razyé II s'est aussi réfugié pour échapper à la colère de son père, ayant partagé avec lui les faveurs de sa maîtresse. Dans cette troisième île et nouvelle mise en scène, la vengeance personnelle de Razyé passe au second plan pour laisser la place à un débat entre Razyé II et Cathy : le premier, fils de son père, croit en la nécessité d'une révolution quelqu'en soit la violence et la deuxième, élevée par Aymeric dans l'admiration de Victor Schœlcher, préconise la tolérance. Elle se laisse pourtant convaincre du paternalisme inhérent aux discours du célèbre abolitionniste et Razyé II, de son côté, se laisse amadouer et, avec l'aide de Cathy, rattrape son éducation interrompue par sa fuite. Leur union pourrait donc représenter une possibilité d'avenir, sauf qu'elle est sans doute incestueuse et qu'ils continuent de fuir Razyé, se réfugiant à la Dominique où Cathy mourra dans l'indigence en mettant au monde Anthuria qui elle, dans la dernière phrase du roman, offre peut-être une lueur d'espoir : « Une si belle enfant ne pouvait pas être maudite » (Condé, 1995, p. 337).

La ré-écriture de Condé nous force à relire l'original et à y découvrir une certaine richesse polyphonique qui n'y paraissait pas avant. La représentation de Heathcliff, au gré du discours de Nelly Dean, est en effet construite à travers plusieurs couches narratives, principalement féminines : en plus des dialogues très succincts qu'elle a avec Mr. Lockwood, Nelly a de longues conversations avec Cathy, Isabelle et la jeune Cathy. Ce geste de solidarité littéraire est aussi un geste de traduction, semblable à celui que Walter Benjamin décrivait dans " la tâche du traducteur ". La traduction assure la survie de l'original tout en le transformant et seuls les textes renfermant le germe de la relation réciproque entre les langues peuvent être traduits. Benjamin, en citant Mallarmé, privilégie la pluralité assurée par la traduction : « Les langues imparfaites en cela que plusieurs, manque la 
suprême : penser étant écrire sans accessoires, ni chuchotement mais tacite encore l'immortelle parole, la diversité, sur terre, des idiomes empêche personne de proférer les mots qui, sinon se trouveraient, par une frappe unique, elle-même matériellement la vérité " (Benjamin, 1997, p. 23). Ainsi, par la ré-écriture, la traduction et l'écriture, Maryse Condé transcende le monde manichéen de Wuthering Heights par une poétique antillaise de l'archipel et souligne la condition déshumanisée du sujet colonial, qu'il soit maître ou esclave. Malheureusement, la traduction anglaise de La migration des cours ne semble pas parvenir à assurer la même survie au roman de Brontë et démontre à ses dépens la fragilité du processus de traduction.

\section{Les eaux troubles de la traduction}

Sa mort nous sépare. Ma mort ne nous réunira pas.

Simone de Beauvoir, La Cérémonie des adieux

Sur le plan métaphorique, la citation de Simone de Beauvoir que Maryse Condé met en deuxième exergue à son roman démontre que le geste de solidarité littéraire effectué par l'écrivaine guadeloupéenne ne prétend pas à l'union harmonieuse des deux auteures. Selon le concept du rhizome, le roman de Condé ne se rattache plus à l'unité du roman de Brontë mais en "fait proliférer l'ensemble » (Deleuze et Guattari, 1980 , p. 15) ${ }^{12}$. Comme on le verra, il semble que la traduction anglaise de La migration des caurs impose à nouveau, bien qu'implicitement, Wuthering Heights comme un point de comparaison. Or, " il n'y a pas de points ou de positions dans un rhizome, comme on en trouve dans une structure, un arbre, une racine. Il n'y a que des lignes " (Deleuze et Guattari, 1980, p. 15). En modulant le texte de Brontë, Condé en avait révélé les lignes et souligné la multiplicité. Son texte nous renvoie au texte anglais mais, à l'instar d'une interprétation musicale s'ouvrant sur l'improvisation, lui rend hommage tout en créant quelque chose de nouveau. Cet acte de transposition, et la re-lecture qu'il précipite, vient aussi déranger la confortable canonicité de l'original : le texte de Brontë est décentré et laisse transparaître la pluralité périphérique dont

\footnotetext{
${ }^{12}$ Il est à noter que, bien que le titre original de La Cérémonie des adieux soit préservé dans la trađuction de l'exergue, la seconde référence à de Beauvoir dans le titre de deux chapitres disparaît dans le choix de traduction de " The Farewell Ceremony ».
} 
il était lui-même issu, mais que l'institution littéraire, en élevant le roman au rang de chef-d'œuvre, a rendue floue. Il est donc regrettable que la traduction anglaise de La migration des cceurs, en invitant une comparaison malaisée entre Brontë et Condé, risque d'être critiquée pour n'être pas à la hauteur de son modèle.

Telle est en effet la teneur d'une recension acerbe de la plume d'une spécialiste en études brontëennes, selon laquelle la " transposition " de Wuthering Heights est " one of the worst translations into English this reviewer has ever read [...] [p]hrases that are idiomatic in modern English are thrown into contexts where they sound ridiculous "; l'auteure parle aussi de " alien history ", de " confusing story ", de " profusion of characters, all with difficult names " et conclut que " in the book's present English translation, and with the added burden of comparison with one of the most beautifully written books in English, Windward Heights offers little sustenance to the English reader " (Fermi, 1998, p. 208). Il est injuste, bien sûr, de critiquer une traduction sans se référer à l'original, mais l'interdit que jette cette critique sur tout effort de transposition vaut la peine d'être examiné de plus près dans la problématique qui nous occupe ici puisque Fermi semble bizarrement regretter que le traducteur n'ait pas suffisamment " naturalisé » le roman de Condé afin de le rapprocher de l'original de Brontë. La résistance que la critique exprime est motivée par le sens d'aliénation provoqué par la ré-écriture de Condé et trahit un refus de permettre au texte de Brontë d'être défamiliarisé ou " déterritorialisé », pour reprendre le terme de Deleuze et Guattari. Cette transformation est ressentie comme inadmissible parce que les textes de Brontë et de Condé se trouvent à jamais liés dans une dynamique parallèle qu'explique à merveille la métaphore filée par les philosophes français :

L'orchidée se déterritorialise en formant une image, un calque de guêpe; mais la guêpe se reterritorialise sur cette image. La guêpe se déterritorialise pourtant, devenant elle-même une pièce dans l'appareil de reproduction de l'orchidée; mais elle reterritorialise l'orchidée, en en transportant le pollen. La guêpe et l'orchidée font rhizome, en tant qu'hétérogènes. On pourrait dire que l'orchidée imite la guêpe dont elle reproduit l'image de manière signifiante (mimesis, mimétisme, leurre, etc.)... En même temps il s'agit de tout autre chose : plus du tout imitation, mais capture de code, plus-value de code, augmentation de valence, véritable devenir, devenir-guêpe de l'orchidée, devenir-orchidée de la guêpe, chacun de ces devenirs assurant la déterritorialisation d'un des termes et la reterritorialisation 
de l'autre, les deux devenirs s'enchaînant et se relayant suivant une circulation d'intensités qui pousse la déterritorialisation toujours plus loin. (Deleuze et Guattari, 1980, p. 17)

Le pollen pris dans l'orchidée du Yorkshire et transporté à la Caraïbe permet la production d'une orchidée antillaise qui ressemble, certes, à l'anglais, mais dont il est aussi irrémédiablement indépendant. Il s'agit donc, à l'aide de quelques exemples, de voir en quoi la traduction effectuée par Richard Philcox assure la continuation de cette dynamique ou, au contraire, menace de l'arrêter.

Le premier reproche à faire au traducteur porte sur le titre : pourquoi avoir choisi un titre si proche de celui de Brontë? Le beau titre de Condé ne laissait en rien deviner, au premier abord, qu'il s'agissait d'une ré-écriture et avait pour mérite d'évoquer deux thèmes familiers aux lecteurs de Condé : l'amour et la migration, ce dernier étant aussi un lieu commun du contexte antillais. Il est bien sûr impossible de savoir quel a été le rôle respectif joué par les éditeurs dans le choix des titres, mais il semble que Love Migrations aurait mieux fait l'affaire, en marquant la distance que Condé a voulu maintenir entre son modèle et les variations qu'il lui a inspirées. En raison de l'association immédiate que le titre établit avec Wuthering Heights, l'allusion contenue dans Windward Heights aux " Windward Islands " passe aussi quasiment inaperçue ${ }^{13}$. De plus, l'emploi forcé du titre dans le texte pour désigner le lieu où réside Hubert Gagneur cause certaines maladresses puisque Condé avait choisi L'Engoulvent comme équivalent de la maison de Wuthering Heights, qu'elle décrit de la façon suivante :

[...] l'Engoulvent, une maison de géreur à moitié en ruine qui s'élevait à Grands-Fonds-les-Mangles sur un plateau calcaire, la "savane désolée ». L'Engoulvent, on l'avait baptisée comme cela, parce que les vents venus du fin fond de l'horizon semblaient s'y engouffrer après avoir tournoyé parmi les calcaires, les cactus cierges et les razyés. On mesurait leur force à l'inclinaison des rares arbres qui poussaient, rabougris, racornis et tordus comme des vieux corps. Quand il y avait cyclone ou tout bonnement tempête ou onde tropicale, c'est comme si des centaines de chevaux étaient lâchés,

\footnotetext{
${ }^{13}$ Il est vrai que, comme le mentionne Roger Little, cette allusion est erronee en ce qui concerne la Guadeloupe qui, strictement parlant, appartient aux «Leeward Islands » (Little, 1999, p. 13).
} 
piaffaient et rugissaient. La mer sortait depuis La Désirade, se gonflait et inondait tout le plateau. (MC, p. 25)

L'équivalence avec le texte de Brontë se réduit à la désolation de l'endroit et il s'avérerait oiseux de vouloir établir des parallèles entre les détails des deux paysages en raison des différences marquées qu'ils contiennent aussi. Les quelques pertes que l'on relève dans la traduction pourtant contribuent à l'effacement subtil de ces différences :

[...] l'Engoulvent, an overseer's house almost in ruins at GrandFonds-les-Mangles [sic], situated on the Windward Heights. It was called l'Engoulvent because the winds seemed to rush in from the horizon, sweeping over the limestone bluffs, the columnar cactus and the heath. You could tell the force of the wind by the way the bent trees grew, stunted and shrivelled like old folk. When there was a hurricane or simply a storm or tropical depression, it was as if hundreds of wild horses had been let loose in a howling stampede. The sea came over from La Désirade, swelled up and flooded the Heights (WH, p. 18).

Le maintien du nom de l'Engoulvent, allié au besoin de rappeler le titre, cause une confusion de nomenclature qui ne semble pas nécessaire, à moins de vouloir faire écho à Wuthering Heights. La perte de la description de Grands-Fonds-les-Mangles est plus difficile à expliquer : bien que la désignation de " plateau » soit compensée plus loin dans " limestone bluffs », "la savane désolée ", que les guillemets désignent comme une appellation locale, disparaît. La perte du qualificatif " rares " pour les arbres efface aussi une autre particularité du paysage antillais. De plus, bien que la traduction de "razyés " par " heath » soit acceptable $e^{15}$, le terme évoque néanmoins le paysage des landes anglaises plutôt que les herbes antillaises et son choix ne semble que motivé par le besoin de faire allusion à Heathcliff. Cette hypothèse

${ }^{14}$ Dans l'analyse qui suit, les références à La migration des cours et à Windward Heights auront recours respectivement aux abréviations $\mathrm{MC}$ et WH.

${ }^{15}$ La définition de " heath " que donne le Oxford English Dictionary Online étant la suivante : "Open, uncultivated ground; an extensive tract of waste land; a wilderness; now chiefly applied to a bare, more or less flat tract of land, naturally clothed with low herbage and dwarf shrubs, esp. with the shrubby plants known as heath, heather or ling ". Il est pourtant mentionné que la plante du même nom est native du sud-ouest de l'Angleterre. 
est d'ailleurs confirmée dès le début du roman lorsque Razyé se présente à Melchior dans les termes suivants :

Je dis « chez moi » pour parler comme tout le monde. Mais je n'ai pas de pays. C'est en Guadeloupe qu'on m'a trouvé nu comme un ver et braillant plus fort qu'un cochon qu'on égorge, en plein milieu des razyés. Mon nom vient de là. $(\mathrm{MC}, \mathrm{p} .17)$

Passage que Philcox traduit ainsi:

I say « home " to speak like the rest of you. But I have no home. I was found in Guadeloupe as naked as the day I was born, on the barren heath and cliffs - the razyés - hence my name. (WH, p. 9)

Si la paraphrase en apposition est justifiable, à cause de la nécessité d'expliquer le terme emprunté de razyé, l'inclusion de cliffs, allusion directe au héros de Brontë, ne l'est pas : la côte de l'île principale de la Guadeloupe, surtout la partie où se trouvent les champs de canne sur lesquels Razyé déversera sa vengeance, ne comprend que peu de falaises et on utilise plus volontiers le terme de morne pour décrire les hauteurs. On se demande aussi pourquoi le traducteur a choisi de passer sous silence le fait que Razyé " braillait ", ce qui pourrait être interprété comme un signe précurseur de sa force de résistance et de sa nature violente. La perte de " pays ", clin d'œil anachronique faisant allusion au statut de DOM de la Guadeloupe, est compréhensible vu la difficulté inhérente au terme de home, qui est pourtant la meilleure traduction possible de « chez moi ».

Ce rapprochement délibéré avec le roman de Brontë n'est pourtant pas conséquent avec d'autres choix de traduction sur lesquels il vaut la peine de s'attarder. Il s'agit en particulier de l'insertion de termes et d'expressions créoles dont Condé parsème son texte. L'analyse révèle un procédé plutôt $a d$ hoc dans la traduction, sans que les décisions prises paraissent toujours justifiées. Contrairement à une pratique établie depuis longtemps par Condé, ou par ses éditeurs, $L a$ migration des cours ne contient aucune note de bas de page et les insertions créoles, ainsi que les emprunts espagnols et africains, bien que mis en italiques, ne sont pas paraphrasés ni expliqués. La version anglaise n'italicise pas ces termes de façon systématique et a parfois recours à des paraphrases en apposition qui ne semblent pas toujours judicieuses. 
Par exemple, " la calle de Mercaderes " (MC, p. 14) devient " the calle de Mercaderes " (WH, p. 7) et " bata-zindien " (MC, p. 15) " Indian half-caste, the Bata-Zindien " (WH, p. 7). L'emploi fréquent de marqueurs créoles offre une autre façon à Condé d'illustrer la distance qui sépare son texte de celui de Brontë, mais pour la version anglaise, les choses se compliquent. Le lecteur anglophone, déjà interpellé par le titre, $s^{\prime}$ attend à plonger dans un récit familier et perd vite pied sur le sol antillais. D'un côté, on pourrait donc s'attendre à ce que la traduction lui facilite la tâche, au moyen d'un glossaire par exemple, vu que le créole à base de français lui est forcément plus étranger qu'au lecteur français. D'un autre côté, le traducteur ne veut pas trahir l'entreprise créolisante de l'auteure et sauvegarde l'étrangeté de son texte, au risque d'aliéner le lecteur. En oscillant ainsi entre deux prérogatives, il se rend vulnérable aux critiques des deux côtés et sa traduction est à la fois trop familière et pas assez. En effet, s'interroge-t-on, pourquoi rendre à la fois explicite le fait que, en tant que $d a$, Nelly est une nursemaid et, dans la description qui suit, effacer tout référent antillais à la couleur de sa peau et à son habillement?

C'est alors que, tandis qu'il cherchait sa cabine dans tout ce désordre, Razyé se heurta à une femme d'âge mûr, belle négresse assez forte et habillée comme une da de bonne famille. Sous la robe matador à ramages, un jupon de dentelles s'arrêtait juste au-dessus de pantoufles de velours violet brodées de fleurs argentées. (MC, p. 24; je souligne)

La raison pour laquelle la proposition qui ouvre ce passage est supprimée dans la traduction n'est pas claire non plus :

Razyé collided with a stout, handsome, middle-aged woman, dressed like a $d a$, a nursemaid from a respectable family, in a flowery, loosefitting dress over a lace petticoat that stopped short above a pair of mauve velvet slippers embroidered with silver flowers. (WH, p. 17; je souligne)

Il n'est pas toujours facile de distinguer, parmi les autres exemples dans cette catégorie, pourquoi certains mots créoles sont retenus et d'autres effacés. Dans le discours direct, les expressions créoles sont maintenues de façon systématique, à quelques exceptions près, sans que le contexte ne vienne nécessairement aider à leur compréhension. L'effet est pour le moins dépaysant et il est regrettable qu'on n'ait pas eu recours à des traductions entre parenthèses ou à des notes en bas de page plus souvent, d'autant plus que cette stratégie est 
utilisée une fois : « Mi bel ti moun, mi! » (MC, p. 275) devenant « Mi bel ti moun, mi! (What a lovely little boy!) " (WH, p. 283). Cette aide apportée au lecteur est incongrue puisqu'elle apparait vers la fin $\mathrm{du}$ texte et qu'elle n'a aucune suite logique avec certaines décisions prises auparavant, notamment celle de traduire une réplique faite en français en rétablissant le créole: « Kimafoutiyesa! Ti-ma-fi, sé on vant a krédi, ou vlé poté ban mwen? E épi yon nèg anko? " (WH, p. 26) pour «Kimafoutiyesa! Ma fille, qu'est-ce que tu veux? Un ventre à crédit? Et avec un nègre encore! " (MC, p. 33). Il est évident que ce passage est difficile à déchiffrer pour tout lecteur non-créolophone, un fait dont Condé est parfaitement consciente.

En ce qui concerne les insertions créoles dans le discours indirect, elles sont aussi traitées de façon inégale, parfois maintenues, disparaissant parfois dans des termes anglais et donnant lieu ailleurs à des traductions différentes. Tel est le cas du terme bonda, effacé lors de son premier emploi et restitué par la suite : "en frétillant du bonda " (MC, p. 29) est traduit par " shaking his behind " (WH, p. 22) alors que, plus loin, « remuait son bonda » (MC, p. 42) donne " wiggled her bonda " (WH, p. 35). Un autre exemple concerne le nom de l'arbre malanga, traduit une fois par " malanga leaves" (WH, P. 25) et une autre par « eddoe " (WH, p. 98). Étant donné la teneur surnaturelle de la relation que Razyé souhaite maintenir avec Cathy outre-tombe, on aurait pu s'attendre à ce que les termes se référant aux esprits malfaisants qui peuplent l'imaginaire antillais soient maintenus dans leur forme originale. Or, c'est loin d'être toujours le cas, au point de mener parfois à de réelles maladresses : « the people in league with the devil had folded up their skins » (WH, p. 279) n'arrive pas, de quelque façon qu'il soit, à rendre le référent culturel des jan gagé, ces êtres surnaturels qui quittent leur peau humaine la nuit pour s'envoler dans la campagne.

Finalement, il reste à mentionner le malaise que cause la traduction du mot nègre, terme à l'emploi très fréquent dans la prose antillaise en français et qui a son équivalent dans le terme créole de nèg, dont un des sens est simplement être humain, mais dont la traduction en anglais est loin d'être évidente ${ }^{16}$. Les exemples que j'ai

\footnotetext{
${ }^{16}$ Le dictionnaire du créole guadeloupéen donne « nègre, homme, cher ami, gars " (Ludwig et al, 1990, p. 236). Pour une analyse plus approfondie que je ne ferai pas ici des problèmes associés avec la traduction de nègre pour un public américain, voir Malena, 1997.
} 
relevés font preuve de l'absence d'une marche à suivre claire et qui s'appliquerait à tout le roman. Le terme, rarement maintenu en créole, est soit effacé par l'équivalent politiquement correct de black, soit inexplicablement renforcé par l'emploi du terme Negroe et même Nigger. La disparition de l'expression créole de nèg-mowon dans le passage suivant semble difficilement justifiable en raison du maintien d'un autre terme créole : " A kind of Soubarou, a wild man of the forest, a runaway slave " (WH, p. 61). L'original était : « Une sorte de Soubarou, de nèg-mawon " (MC, p. 67). Cette traduction, d'autant plus douteuse vu que plus loin le terme est rendu par Maroons (WH, p. 174), ne garantit en rien l'interprétation du lecteur qui pourrait tout aussi bien être celle d'une énumération qu'une double explication de Soubarou. Le recours fréquent aux euphémismes a pour effet de neutraliser la teneur raciale du texte, notamment lorsque nègre est remplacé par black (MC, pp. 28, 73, 181, 195, 215, 253, 289; WH, pp. $21,67,181,194,217,259,297)$. À de rares occasions, cette stratégie a un côté positif comme, par exemple, lorsqu'il s'agit de décrire avec une pointe d'ironie le cortège funèbre d'Aymeric de Linsseuil, " le modèle du bon Blanc, du bon patron ", qui défile le long de la rue du PèreLabat :

Derrière lui [l'orchestre], des dizaines de garçonnets et de fillettes portant des gerbes de fleurs blanches, des petits Blancs-pays, enfants de sa parentèle ou de ses amis. Mais aussi des petits nègres, les enfants de ses ouvriers ou bien de l'école de son domaine et des petits mulâtres. Enfin, ce n'était pas affaire de couleur. (MC, p. 242)

Dans ce passage, la traduction de petits nègres par little black children ajoute à l'ironie qui souligne le comportement paternaliste d'Aymeric, grand admirateur de Victor Schœlcher (WH, p. 247).

D'un autre côté, la stratégie de l'euphémisme va parfois trop loin et finit par effacer l'idée de couleur qui est pourtant au centre du contexte socio-historique peint par Condé. Ainsi, " un cortège de nègres en haillons " devient " a procession of men in rags " (MC, p. 137; WH, p. 133), « les nègres les plus dociles " sont « the most docile of men » (MC, p. 244; WH, p. 240) et « [q]uelques nègres et négresses dépareillés " se réduit à " [a] few motley men and women " (MC, $\mathrm{p}$. 102; WH, p. 98). Plus grave encore, ce genre d'effacement a le potentiel de nuire à la compréhension du lecteur lorsque la mention d'un nègre nago est rendue par Nago, terme pas nécessairement déchiffrable comme désignant une origine africaine en l'absence du référent (MC, p. 121; WH, p. 118). Par contraste avec cette politique 
d'effacement, les quelques exemples où le traducteur choisit de marquer la teneur raciale de l'original sont difficilement acceptables, pour ne pas dire choquants.

Passe encore l'adoption du terme negro(es) (MC, pp. 13, 71, 227; WH, pp. 13, 77, 229), choix justifié dans le contexte de la période qui suit l'abolition de l'esclavage ${ }^{17}$, mais quelle logique incite à marquer la connotation négative associée à Hubert Gagneur et à l'effacer en ce qui concerne Cathy, sa fille? En français, le mulâtre " injuriait comme un nègre des bois » et en anglais, il « swore like a field nigger » alors qu'en français, « Cathy dansait comme une négresse des plantations 》 et « danced like a field girl » en anglais (MC, pp. 28, 29; WH, pp. 22, 23). Une autre instance de nigger survient dans la bouche d'un blanc, cousin d'Aymeric et personnage mineur :

Moi, comme tu me vois là, je n'ai de blanc que la couleur de ma peau. Je mange comme un nègre; je charroie mon rhum comme un nègre; $\mathrm{j}$ 'injurie et je me bats comme un nègre; quant à baiser, je baise comme un nègre, et c'est pour cela qu'Alicia ne peut pas me supporter. (MC, p. 182)

The only white you see in me is the colour of my skin. I eat like a nigger; I swig my rum like a nigger; I swear and I fight like a nigger; as for fucking, I fuck like a nigger, and that's why Alicia can't stand me. (WH, p. 182)

La grossièreté du personnage et sa force métaphorique en tant que représentant de la déchéance de la société coloniale ne sont pas à remettre en question, mais il semble qu'il eût été possible de les marquer de façon tout aussi acceptable dans la traduction des verbes, tel qu'il a déjà été fait dans le cas de baiser. Bien que Windward Heights ait été publié en Angleterre, il est évident qu'il sera lu par des lecteurs américains pour qui le terme nigger évoque des événements douloureux beaucoup plus récents que l'esclavage. Un dernier exemple concerne la traduction de mal-nèg, toujours utilisé en rapport avec Razyé à cause sans doute de son évocation des mauvais esprits, qui donne lieu une fois à nigger stud et deux autres à « one hell of a man » (MC, pp. 78, 268, 275; WH, pp. 72, 275, 282). Là aussi, la traduction apparaît comme trop forte ou pas assez, l'idée de puissance sexuelle

${ }^{17}$ Voir Kadish (1994, p. 19) pour une rationalisation de l'emploi du terme dans la traduction de textes à teneur abolitionniste. 
étant suffisamment bien rendue par stud dans le premier exemple et disparaissant complètement dans les deux suivants. Le maintien du terme créole dans les deux cas aurait évité le problème. Dans l'ensemble, l'analyse de la traduction anglaise de La migration des cours révèle donc des stratégies ad hoc qui brouillent les pistes d'interprétation et mènent à s'interroger sur le but envisagé par la parution de Windward Heights : s'il s'agissait de rapprocher le texte de Condé de celui de Brontë, comme le suggère d'emblée le choix du titre, pourquoi dépayser le lecteur par le maintien fréquent de passages en créole sans explication quelconque? D'un autre côté, s'il s'agissait de respecter le mouvement isentropique et créateur du texte de Condé, pourquoi en effacer les marqueurs du contexte socio-historique antillais?

En conclusion, La migration des caurs s'inscrit dans une logique d'adaptation qui n'est pas sans rappeler la théorie de « Woman Version » élaborée par Evelyn O'Callaghan (1993). Par le truchement d'un processus métonymique de traduction, Maryse Condé interprète l'ouvrage classique d'Emily Brontë en le modulant au rythme et aux sons antillais. O'Callaghan insiste sur la complexité de cette forme de " sub/version " qui naît " out of a process of altering, supplementing, breaking, echoing, mocking and playing with that original $1{ }^{18}$ (O'Callaghan, p. 11). Comme le rappelle Maria Tymoczko, « a literary work, like a translation, depends on previous texts: neither is an "original semantic unity", both are "derivative and heterogeneous" [...] Every writing is a rewriting " (Tymoczko, 1999, p. 41). Le travail de ré-écriture auquel se livre Condé, et qui s'apparente au travail de traduction, souligne la relation métonymique que sa création entretient avec d'autres cuvres mais supporte mal une re-traduction dans le sens du modèle original. En voulant donner à Wuthering Heights une nouvelle voix, Condé faisait beaucoup plus que simplement en proposer une nouvelle version : elle racontait une nouvelle histoire, dans le sens d'Histoire et de récit, et elle ré-interprétait le drame déroulé sur les landes du Yorkshire en l'inscrivant dans le violent contexte socio-historique de la Caraibe. La version anglaise de son texte risque d'être mal reçue parce qu'il manque au public anglais la

\footnotetext{
${ }^{18} \mathrm{~J}$ 'adapte ici les propos de O'Callaghan qui portent sur les stratégies d'écriture féminine aux Antilles en réaction contre le discours dominant masculin. Il est vrai, comme le mentionne Françoise duRivage, que le geste de solidarité effectué par Condé envers Brontë " s'inscrit aussi dans une tradition qui justement s'oppose au modèle masculin européen " (duRivage, 1999, p. 22).
} 
familiarité nécessaire avec l'histoire et la culture des Antilles françaises pour pouvoir accepter cette transposition d'un classique favori (voir Tymoczko, 1999, p. 47). Doit-on alors simplement en conclure à un échec et faire écho au "The subaltern cannot speak " de Spivak (Spivak, 1994, p. 104)? J'espère avoir montré que la réponse à cette question se doit d'être négative puisque, malgré les problèmes associés à sa traduction en anglais, dont plusieurs sont remédiables, l'ouvrage de Condé est une performance littéraire réussie qui déconstruit le discours impérialiste. Par le fait d'une transposition contextuelle et linguistique, La migration des cours révèle la violence inhérente à un régime colonial dont le pouvoir est assuré par le désir de constituer le sujet antillais comme l'Autre absolu de l'Européen (voir Spivak, 1994, p. 76). En multipliant les narrateurs et les personnages et en jouant sur la focalisation, Condé illustre la non-réalisation de ce désir, la complexité de la situation coloniale, la nature inextricable de la relation entre sujet colonisé et sujet colonisateur, leur hétérogénéité respective jamais totalement déchiffrable et, par contre-coup, les aspects " coloniaux » du texte de Brontë. Cette migration littéraire implique que l'écriture s'appuie sur des procédés de traduction et que la ré-écriture maintient une difficile relation métonymique avec l'original en lui rendant hommage tout en le transformant.

Université de l'Alberta 


\section{DOMAINE DE THRUSHCROSS GRANGE}

Mr. et Mrs. Linton

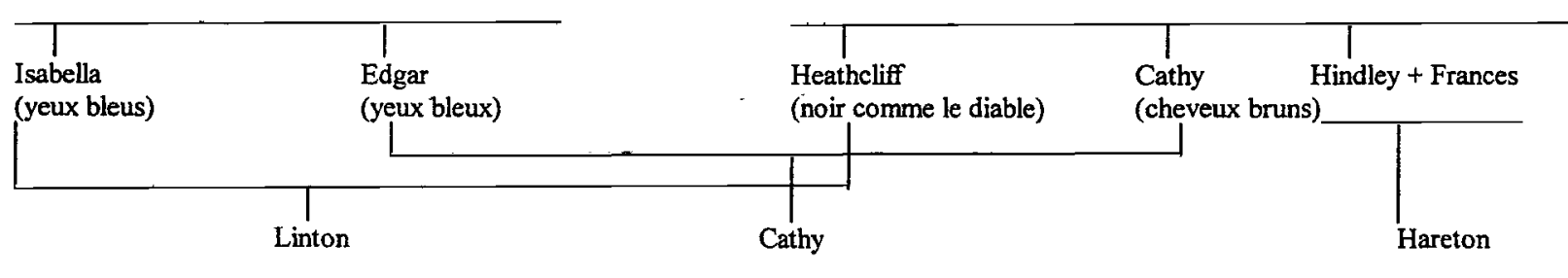

LES GENEALOGIES DE LA MIGRATION DES COEURS

DOMAINE DES BELLES-FEUILLES

De Linsseuil

(Béké)

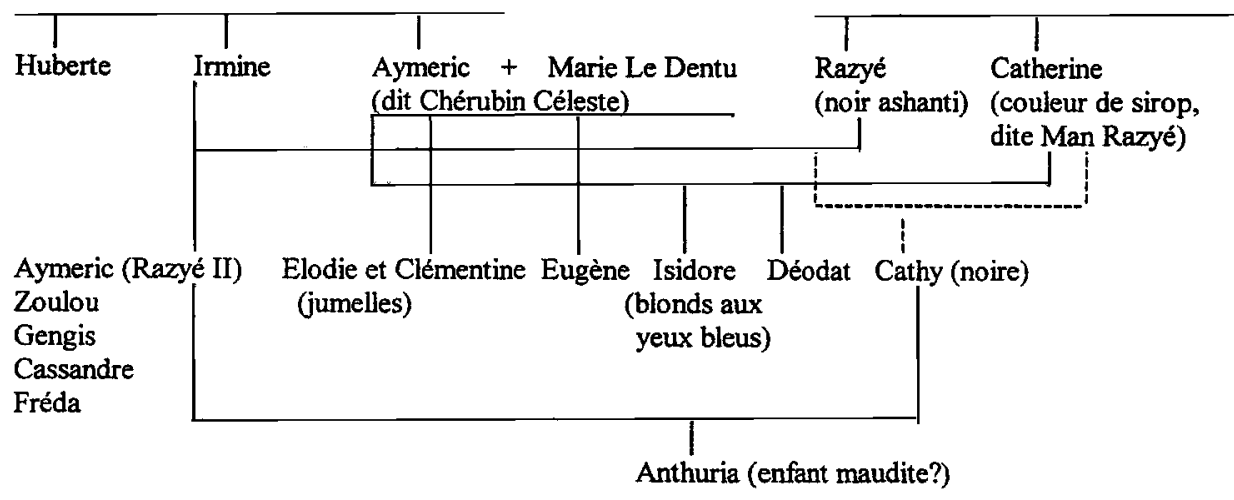

DOMAINE DE L'ENGOULVENT

Hubert Gagneur + Irminette Boisgris (mulâtre)

$$
\begin{aligned}
& \text { Justin + Marie-France } \\
& \text { (peau claire) La Rinardière } \\
& \text { (parente des de } \\
& \text { Linsseuil, tuber- } \\
& \text { culeuse) } \\
& \text { Justin-Marie } \\
& \text { (poitrinaire) }
\end{aligned}
$$




\section{Références}

BENÍTEZ-ROJO, Antonio (1992). The Repeating Island. Traduit par James E. Maraniss. Durham and London, Duke University Press.

BENJAMIN, Walter (1997). « L'abandon du traducteur. Prolégomènes à la traduction des Tableaux Parisiens de Charles Beaudelaire "). Trad. Laurent Lamy et Alexis Nouss. TTR, vol. X, n² 2, pp. 13-69.

BRONTË, Charlotte (1960). Jane Eyre. New York and Scarborough, New American Library, A Signet Classic.

BRONTË, Emily (1992 [1929]). Les Hauts de Hurle-Vent. Traduit par Frédéric Delebecque. Paris, Payot.

- (1959; [1847]). Wuthering Heights. New York and Scarborough, New American Library, A Signet Classic.

CONDÉ, Maryse (1989). Traversée de la mangrove. Paris, Mercure de France.

— (1995). La Migration des caurs. Paris, Robert Laffont.

- (1998). Windward Heights. Traduit par Richard Philcox. London, Faber and Faber.

DELEUZE, Gilles et Félix GUATTARI (1980). Mille plateaux : capitalisme et schizophrénie. Paris, les Éditions de Minuit.

DuRIVAGE, Françoise (1999). " Réécriture de la folie dans $L a$ migration des cours de Maryse Condé ». Romance Languages Annual $\mathrm{X}, \mathrm{pp} .22-27$.

EAGLETON, Terry (1992). " Myths of Power: A Marxist Study of Wuthering Heights ». Emily Brontë. Wuthering Heights. Ed. Linda H. Peterson. Boston, St.Martin's Press, pp. 399-414.

FAULKNER, William (1987 [1930]). As I Lay Dying. New York, Vintage Books.

FERMI, Sarah (1998). « Book Review of Windward Heights ». Brontë Society Transactions, vol. 23, n 2, pp. 207-208. 
HUGO, Victor (sd). Bug-Jargal. Paris, J. Hetzel.

KADISH, Doris Y. and Françoise MASSARDIER-KENNEY, eds. (1994). Translating Slavery: Gender and Race in French Women's Writing, 1783-1823. Kent, OH, The Kent State University Press.

LITTLE, Roger (1999). " Condé, Duras, Beyala: Intertextuality or Plagiarism? ». French Studies Bulletin 72, pp. 13-15.

LUDWIG, Ralph, Daniele MONTERRAND, Hector POULLET, Sylviane TELCHID (1990). Dictionnaire Créole Français (Guadeloupe). Servedit/Éditions Jasor, 1990.

MALENA, Anne (1997). « En anglais, Léonora parle-t-elle encore et à qui? ». TTR, vol. $\mathrm{X}, \mathrm{n}^{\circ} 2$, pp. 197-218.

- (1999). The Negotiated Self: The Dynamics of Identity in Francophone Caribbean Narrative. New York, Peter Lang.

O'CALLAGHAN, Evelyn (1993). Woman Version: Theoretical Approaches to West Indian Fiction by Woman. London and Basingstoke, McMillan Press.

PEIRCE, Charles Sanders (1977). Semiotics and Significs. Ed. Charles S. Harwick. Bloomington, Indiana University Press.

PETERSON, Linda H. (1992). " Biographical and Historical Contexts ». Emily Brontë. Wuthering Heights. Ed. Linda H. Peterson. Boston, St. Martin's Press, pp. 1-12.

RHYS, Jean (1968). Wide Sargasso Sea. Harmondsworth, Penguin Books Ltd.

SPIVAK, Gayatri Chakravorty (1985). "Three Women's Texts and a Critique of Imperialism». Critical Inquiry, vol. 12, $\mathrm{n}^{\circ}$ 1, pp. 243-261.

- (1994). «Can the Subaltern Speak? ». Colonial Discourse and PostColonial Theory: A Reader. Ed. Patrick Williams and Laura Chrisman. New York, Columbia University Press. 
THOMAS, Hugh (1997). The Slave Trade: The History of the Atlantic Slave Trade 1440-1870. London, Picador.

TYMOCZKO, Maria (1999). Translation in a Postcolonial Context: Early Irish Literature in English Translation. Manchester, UK, St. Jerome Publishing.

\section{RÉSUMÉ : Migrations littéraires : Maryse Condé et Emily Brontë}

- En tant que ré-écriture de Wuthering Heights (1847) d'Emily Brontë, La Migration des caurs (1995) de Maryse Condé transpose le classique anglais dans un contexte antillais marqué par la violence colonialiste et l'hétérogénéité. Ce procédé de ré-écriture est un procédé de traduction dans le sens large du terme parce que l'improvisation à laquelle se livre Condé maintient un lien métonymique avec l'original tout en fonctionnant de façon indépendante. À son tour, la traduction anglaise du roman de Condé, Windward Heights (1998), suit ces pistes brouillées mais, par manque de stratégies conséquentes de traduction, compromet l'élan créateur de Condé en rapprochant son texte trop près de celui de Brontë. Cette étude montrera que ces mouvements de migration littéraire impliquent que l'écriture s'appuie sur des procédés de traduction et que la ré-écriture maintient une difficile relation métonymique avec l'original en lui rendant hommage tout en le transformant.

\section{ABSTRACT: Literary Migrations: Maryse Condé and Emily} Brontë - As a rewriting of Emily Brontë's Wuthering Heights (1847) Condé's La Migration des cceurs (1995) transposes the English classic into a Caribbean context characterized by colonial violence and heterogeneity. Rewriting is a translation process in the broad sense of the term since Conde's improvisation remains linked metonymically with the original in spite of functioning independently. Windward Heights (1998) then, the English translation of Condé's novel, follows these intermingling antecedents but threatens to stop Condé's creative drive because it calls for too close a comparison with Brontë's text from lack of consistent translation strategies. This study will show how these literary migrations suggest that writing is akin to a process of translation and that rewriting maintains a difficult metonymic relation with the original, transforming it while acknowledging it. 
Mots-clés : ré-écriture, intertexte, créolisation, colonialisme, stratégies de traduction.

Key words: rewriting, intertext, creolization, colonialism, translation strategies.

Anne Malena : MLCS, 200 Arts Building, University of Alberta, Edmonton AB T6G 2E6

Courriel : amalena@ualberta.ca 